\title{
Magnetic fields and plasmas in the inner heliosphere: Helios results
}

\author{
L.F. Burlaga* \\ Laboratory for Extraterrestrial Physics, NASA/Goddard Space Flight Center, Code 692, Greenbelt, MD 20771, USA
}

Received 7 November 2000; accepted 18 January 2001

\begin{abstract}
The magnetic field of Mercury and the structure and dynamics of Mercury's magnetosphere, which will be studied by the spacecraft orbiting Mercury, are strongly influenced by the interaction of the solar wind with Mercury. In order to understand the internal magnetic field, it will be necessary to correct the observations of the external field for the distortions produced by the solar wind. Understanding of the solar wind interaction with Mercury is essential for understanding the structure and dynamics of the magnetosphere and phenomena such as magnetic storms. Helios 1 and 2 made a number of passes in the region traversed by the orbit of Mercury, and each pass provided a sample of the solar wind environment of Mercury. This paper reviews the plasma and magnetic field observations from Helios that provide a general basis for interpreting the observations of Mercury that will be made by orbiting spacecraft. The variables that govern the structure and dynamics of the magnetospheres of Mercury and Earth are approximately 5-10 times larger at Mercury than at Earth. Thus, the solar wind interaction with Mercury will be much stronger than the interaction with Earth. Moreover, the solar wind at Mercury is probably more variable than that at Earth. There is a clear need for measurements of the solar wind during the approach of spacecraft to Mercury and while they are in orbit around Mercury. (c) 2001 Elsevier Science Ltd. All rights reserved.
\end{abstract}

\section{Introduction}

One of the most important discoveries of the MercuryVenus Mission is that Mercury has a magnetic field (Ness et al., 1974; Ogilvie et al., 1974) and a magnetosphere (Whang, 1977). Models of the magnetic field of Mercury are discussed by Connerney and Ness (1988). Although there are significant uncertainties in knowledge of the magnetic field of Mercury owing to the limited observations, to first approximation one can consider the field to be that of a dipole whose axis is within $\approx 15^{\circ}$ to the normal to the ecliptic. The magnetic field lines on the dayside of Mercury lines leave the southern hemisphere and enter the northern hemisphere just as they do at Earth.

Since Mercury is embedded in the solar wind, the solar wind distorts the planetary magnetic field and it drives dynamical processes in the magnetosphere (Russell et al., 1988). In order to determine the planetary magnetic field accurately, it is necessary to correct for changes produced by the solar wind as the magnetometers move through the region occupied by the planetary magnetic field. In order to understand magnetospheric dynamical processes, it is necessary to understand the solar wind conditions at the orbit of Mercury that drive these processes.

\footnotetext{
${ }^{*}$ Tel.: +1-301-286-5956; fax: +1-301-286-1684.

E-mail address: burlaga@lepvax.gsfc.nasa.gov (L.F. Burlaga).
}

The factors that influence the magnetic field and magnetosphere of Mercury are probably the same as those that influence the magnetic field and magnetosphere of Earth. These are the proton density $N$, the solar wind speed $V$, the solar wind momentum flux which is proportional to $N V^{2}$, the Mach numbers, the strength $B$, direction, and variability of the interplanetary magnetic field, $\boldsymbol{B}$, and the electric field $E=\boldsymbol{V} \times \boldsymbol{B}$ (Baker et al., 1984). Thus, knowledge of $N, B$, and $\boldsymbol{V}$ at the orbit of Mercury is essential for understanding Mercury's magnetic field and magnetosphere. Additional measurements such as the temperatures that influence characteristic speeds and Mach numbers are also important.

The Helios 1 and 2 spacecraft sampled the solar wind conditions near the orbit of Mercury on several orbits. Reviews of the plasma, magnetic field and energetic particle observations made during the Helios missions were published by Schwenn (1990), Mariani and Neubauer (1990), Burlaga (1991), Marsch (1991) and Kunow et al. (1991). The purpose of this invited paper is to review the Helios observations that are relevant to the design of the Mercury orbiter payload and the interpretation of the corresponding particle and field observations at Mercury.

\section{Orbits}

The orbit of Mercury is very eccentric, with an aphelion 0.47 $\mathrm{AU}$ and perihelion 0.31 AU, as illustrated in Fig. 1. 


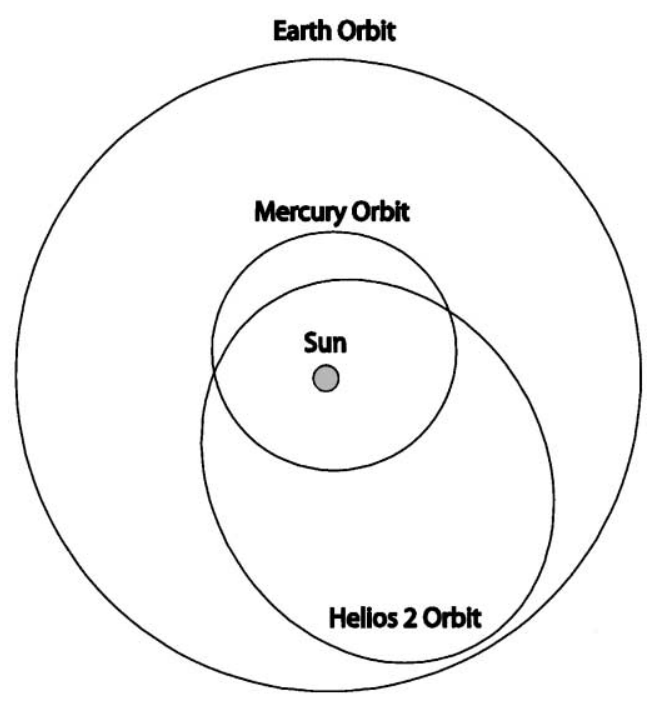

Fig. 1. Orbits of Earth, Mercury and Helios 2. The orbit of Helios 1 (not shown) is similar to that of Helios 2, except for a phase shift of the major axis.

We shall refer to the region between 0.31 and $0.47 \mathrm{AU}$ as "Mercury's orbital zone". The orbit of Helios 2 is an ellipse in the ecliptic with a perihelion of $0.29 \mathrm{AU}$ and an aphelion of $0.98 \mathrm{AU}$, as illustrated in Fig. 1. Thus, Helios 2 passed through Mercury's orbital zone. The orbit of Helios 1 was similar to that of Helios 2, except that its semi-major axis was oriented differently and its perihelion, was $0.31 \mathrm{AU}$. The orbital period of both Helios 1 and Helios 2 was 6 months, and the time to pass through the orbital zone of Mercury was $\approx 1$ month. Each spacecraft passed through the orbital zone twice per year, spending approximately 2 months of each year in Mercury's orbital zone. Helios 1 provided data from 1974 to 1986, but the data coverage was incomplete for many of the passes. Although we have a good sample of the interplanetary conditions near the orbit of Mercury, it is far from being continuous coverage over a solar cycle.

\section{Large-scale structure}

\subsection{Radial variations}

Daily averages of the magnetic field strength measured by Helios 1 during 1975 are plotted as a function of the logarithm of distance $R$ from the Sun in Fig. 2. The horizontal line between the two vertical dashed lines indicates Mercury's orbital zone. Approximately 6024 -h averages are in the orbital zone. The solid curve in the figure is a fit of the data to a second order polynomial. The fit gives $B=6 \mathrm{nT}$ at $1 \mathrm{AU}$, which is close to the average value of $B$ at $1 \mathrm{AU}$ over the solar cycle. The magnetic field strength at the orbit of Mercury is $\approx 3-6$ times the average field strength at $1 \mathrm{AU}$. The daily average fluctuates significantly and can be as large as 8 times the average value at $1 \mathrm{AU}$. Hour averages of $B$ (not shown in Fig. 2) fluctuate by an even greater amount.
Helios 1

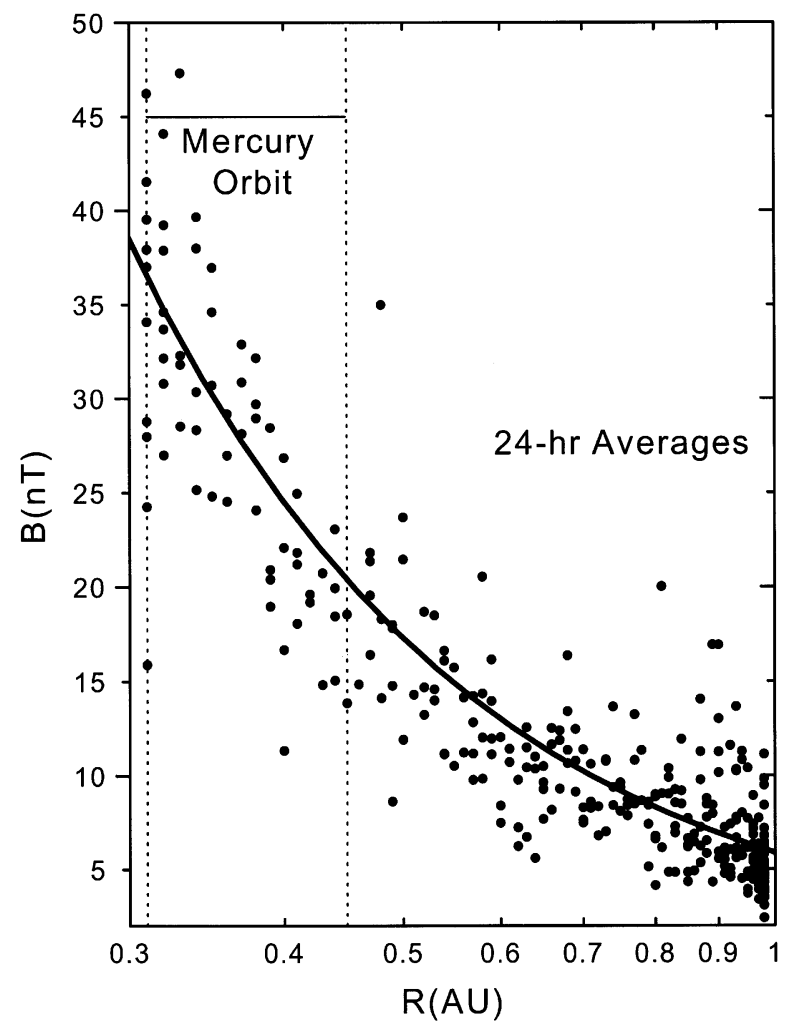

Fig. 2. Magnetic field strength as a function of distance $R$ from the Sun (on a $\log$ scale). The data are 24-h averages of the magnetic field measured by Helios 1 from during its first year of operation, which includes two passes through Mercury's orbital zone.

Daily averages of the solar wind speed measured by Helios 1 during 1975 are shown in the bottom panel of Fig. 3. The solar wind speed does not vary significantly with distance from the Sun between 0.29 and $0.98 \mathrm{AU}$. The mean value of $V$ in this interval is $460 \mathrm{~km} / \mathrm{s}$. This speed is higher than the average speed over a solar cycle $(\approx 400 \mathrm{~km} / \mathrm{s})$, because the measurements were made during the declining phase of the solar cycle, when fast flows from the polar coronal holes extended to the ecliptic. The variability of the daily averages of $V$ is very large during this period, with speeds ranging from 250 to nearly $750 \mathrm{~km} / \mathrm{s}$.

The momentum flux $\left(\propto N V^{2}\right)$, which strongly influences the size of Mercury's magnetosphere, is shown in the top panel of Fig. 3 on a $\log -\log$ scale. The momentum flux is as variable in Mercury's orbital zone as it is at Earth. The momentum flux varies with distance from the Sun as $R^{-(1.95 \pm 0.05)}$. Thus, the momentum flux at Mercury is $\approx$ 5-10 times that at Earth, which implies that the momentum flux influences the magnetosphere of Mercury much more strongly than the magnetosphere of Earth.

As a result of conservation of mass in a radial flow in a spherical geometry, $N V R^{2}$ is a constant. Since $V$ is a constant independent of $R$, one expects the density to vary as $R^{-2}$. Fig. 4 shows that this is the case for the daily averages of 

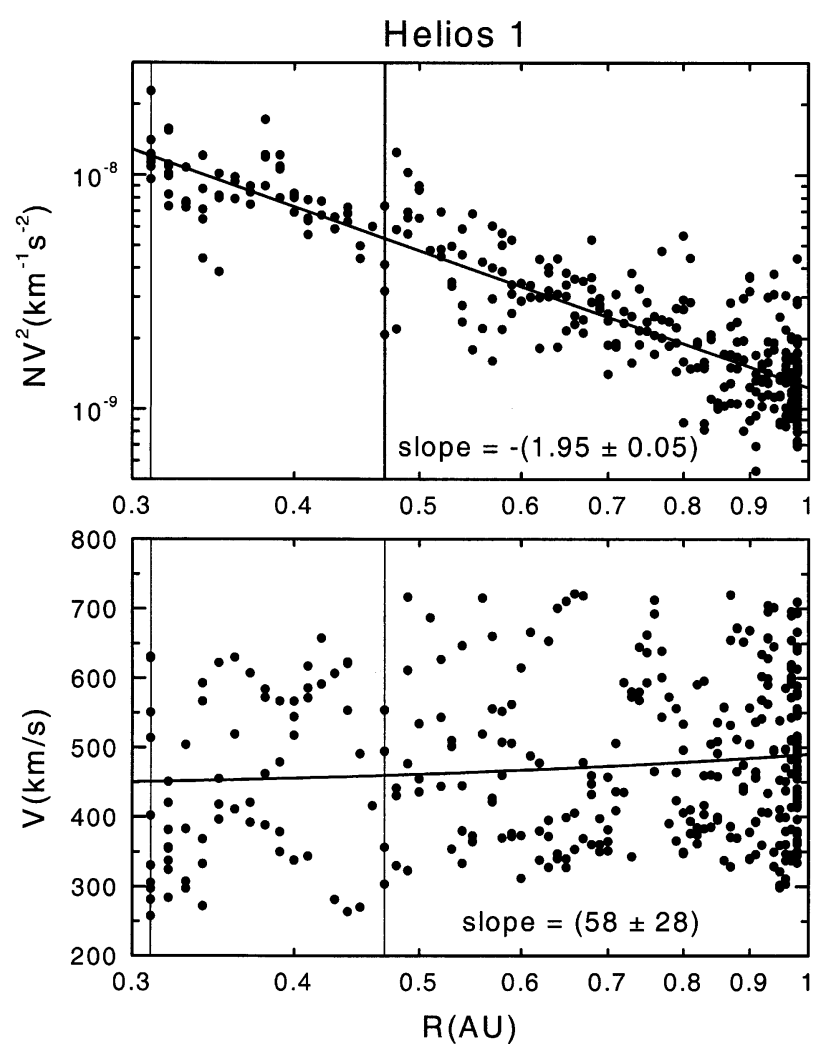

Fig. 3. Top: $N V^{2}$, which is proportional to the momentum flux, as a function of distance $R$ from the Sun. The line is a linear least squares fit. Bottom: The solar wind speed $V$ as a function of distance from the Sun. The density and speed in this figure are 24-h averages.

density measured by Helios 1 during 1975. A linear least squares fit to the data, which are plotted on a log-log scale, gives $N \propto R^{-(2.1 \pm 0.1)}$, which is consistent with Bougeret et al. (1984). The $R^{-2}$ dependence of the momentum flux is a consequence of the radial variation of the density, since $V$ is approximately independent of $R$. Fig. 4 shows that the density at Mercury is $\approx 5-10$ times that at Earth.

The variability of the magnetic field direction has an important effect on the variability of Earth's magnetosphere, and one expects a similar effect at Mercury. The sum of the variances of the magnetic field components, $\sigma_{1}^{2}$, was computed over 3 -h intervals as a function of distance from the Sun (Mariani et al., 1978) for both high speeds $(>600 \mathrm{~km} / \mathrm{s})$ and low speeds $(<500 \mathrm{~km} / \mathrm{s})$. They found that $\sigma_{1}^{2} \propto R^{-3}$ for both high and low speeds. Denskat and Neubauer (1982) obtained similar results. Thus, the RMS variability of the magnetic field is $3-6$ times greater at Mercury than it is as Earth.

\subsection{Distribution functions and long-term averages}

Statistical distributions of hourly averages of Helios 1 and Helios 2 observations made in the region $\approx 0.30-0.47 \mathrm{AU}$ from 1974 (the declining phase of the solar cycle) to 1980 (solar maximum) were published by Russell et al. (1988)
Helios 1

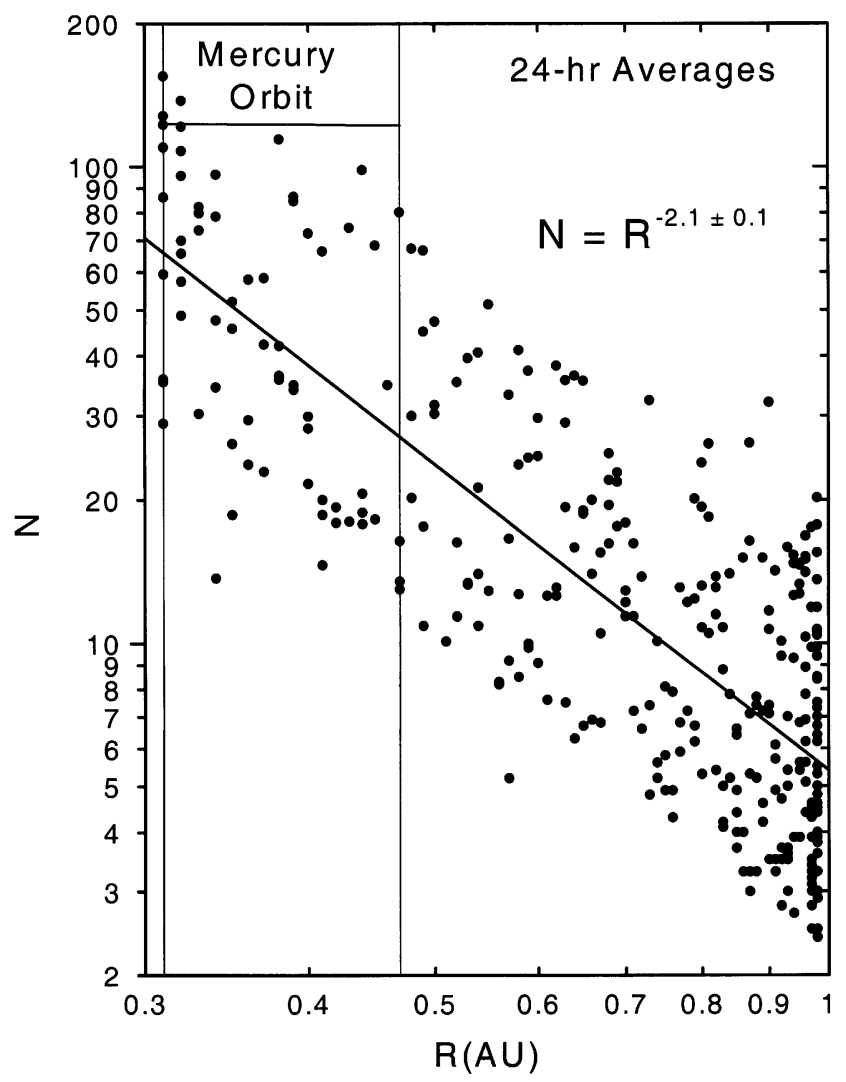

Fig. 4. A $\log -\log$ plot of density as a function of distance from the Sun.

(see also Musmann et al., 1977; Marsch et al., 1982). The means and standard deviations of the distributions of magnetic field strength $B$, density $N$, and bulk speed $V$ are $30.5 \pm 11.2 \mathrm{nT}, 59.8 \pm 44.4 \mathrm{~cm}^{-3}$, and $423 \pm 112 \mathrm{~km} / \mathrm{s}$, respectively for a period of approximately half a solar cycle. On average, the magnetic field strength and density in the orbital zone of Mercury are 5 and 10 times that at Earth, respectively, and the speed is the same as that at Earth, consistent with the Parker spiral field model (Parker, 1963; Slavin and Holzer, 1981). Thus, to extent that the interaction of the solar wind with Mercury depends on $B$ and $N$, it is $5-10$ times as strong as the interaction of the solar wind with Earth.

\section{A sample of the solar wind in Mercury's orbital zone}

Observations made by Helios 1 in the orbital zone of Mercury during its first perihelion pass in March 1975 (Burlaga et al., 1978) are shown in Fig. 5. The top four panels in Fig. 5 SHOW THE MAGNETIC field strength, $B$, speed, $V$, density, $n$, and the proton temperature $T$ as a function of longitude, measured in "Helios Solar Rotations, HSR". Each solar rotation is numbered by an integer (in this case 3 ) and a decimal indicating the fraction of $360^{\circ}$ covered at the time of the observations. Fig. 5 shows that Helios moved 


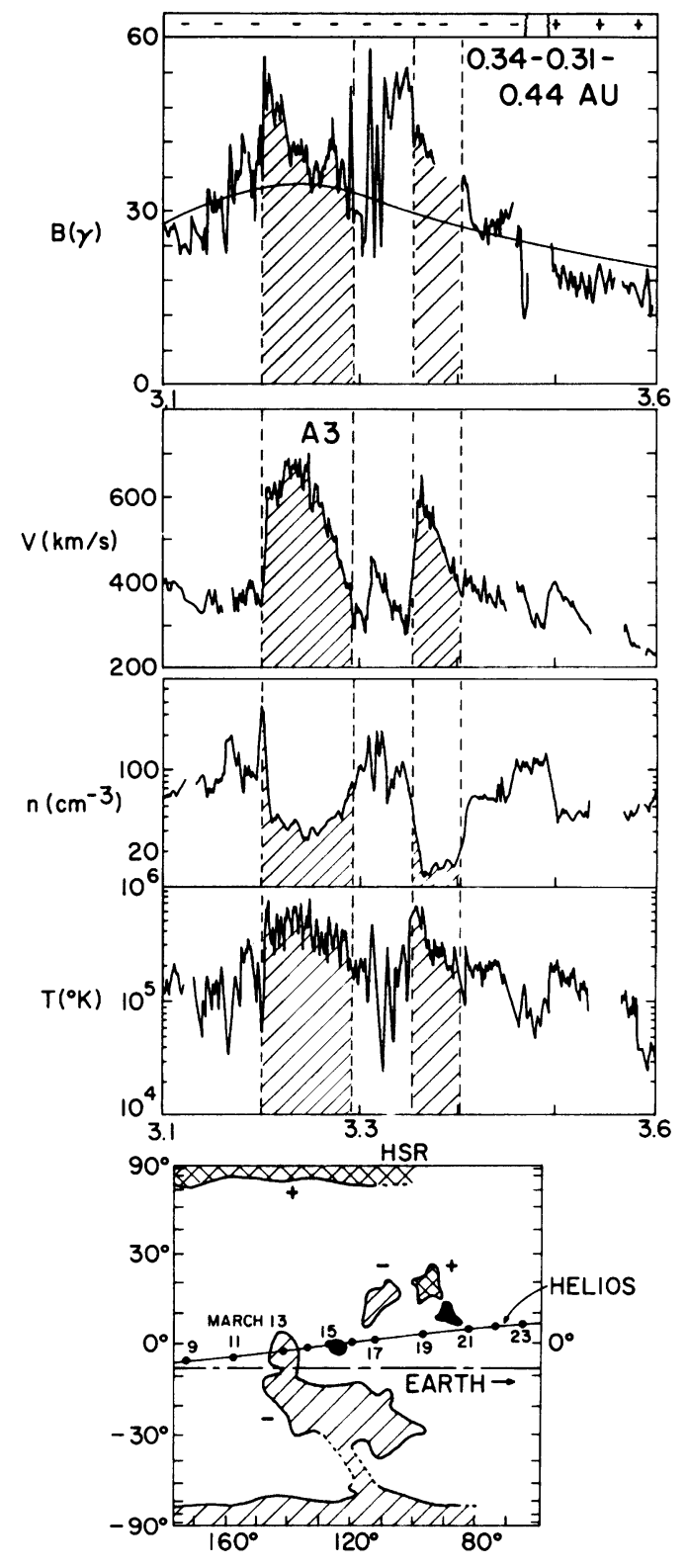

Fig. 5. Observations of the magnetic polarity, magnetic field strength, speed, density and proton temperature. The bottom panel shows coronal holes and the projection of the trajectory of Helios 1 on the Sun.

through $115^{\circ}$ in longitude in $\approx 16$ days as it moved from a radial distance of $0.34-0.31 \mathrm{AU}$ and back out to $0.44 \mathrm{AU}$.

A striking result of this sample of conditions in Mercury's orbital zone is that the environment of Mercury can be highly variable. The magnetic field strength ranges from $\approx 15$ to $\approx 55 \mathrm{nT}$, and the field strength fluctuates considerably. The density shown in Fig. 5 ranges from 18 to $350 \mathrm{~cm}^{-3}$, and there are very abrupt changes by an order of magnitude. The speed profile shows two narrow streams with speeds greater than $600 \mathrm{~km} / \mathrm{s}$ and smaller fluctuations. The proton temperature varies from 20,000 to $700,000 \mathrm{~K}$, and it too shows large and rapid fluctuations. The kinds of fluctuations evident in Fig. 5 will have a strong impact on
Mercury's magnetosphere and hence on the interpretations of measurements made by an orbiter within the magnetosphere. Fig. 5 shows just one brief sample of only one type of flow configuration within Mercury's orbital zone. Studies of Mercury will require a much better understanding of its environment than we can obtain from a few samples such as that in Fig. 5.

\section{Corotating streams}

Corotating streams are quasi-stationary flows that tend to corotate with the Sun and often recur at a point such as Mercury or Earth for one or more solar rotations. Corotating streams have a well-defined signature at $\leqslant 1$ AU: high speeds, high temperatures and low densities following a stream interface, which is characterized by an abrupt decrease in density and abrupt increases in temperature and speed (see, e.g., Hundhausen, 1972; Burlaga, 1974). Fig. 5 shows that the two streams observed by Helios during its first perihelion passage (indicated by the cross-hatch pattern) are corotating streams. The large variations in density and temperature in this interval are primarily associated with the two corotating streams.

Studies based on measurements made at 1 AU have shown that corotating streams originate in coronal holes (see Hundhausen, 1977 for an excellent review and references). The bottom panel of Fig. 5 shows coronal holes measured from Earth in He 10830 during the first perihelion passage of Helios 1. The scale and position of the lower panel in Fig. 5 were chosen to correspond to the magnetic field and plasma measurements shown in the panels above, with a propagation time delay. The projection of the Helios trajectory on the Sun is also plotted in the lower panel of Fig. 5. Helios 1 moved from $0.43 \mathrm{AU}$ on March 9 to perihelion at $0.31 \mathrm{AU}$ and back out to $0.44 \mathrm{AU}$ on March 23. The first corotating stream (A3) in Fig. 5 is associated with passage of Helios above the equatorial extension of the south polar coronal hole. The magnetic polarity in the coronal hole ("--", corresponding to a magnetic field pointing toward the Sun) agrees with the polarity of the interplanetary magnetic field measured by Helios, which is shown at the top of Fig. 5. After Helios moved over an active region (a small dark area in the lower panel) on March 15, it observed slow, high-density, cold material. On March 17, the path of Helios was just south of a near-equatorial coronal hole. The second corotating stream probably originated in this coronal hole, because both the solar field and the magnetic field at Helios had negative polarity and because the stream arrived at Helios with the expected time delay from the passage of the coronal hole beneath it. Helios moved past a near-equatorial hole of positive polarity on March 19 and 20, but it did not observe a corresponding corotating stream. Perhaps the absence of a stream is due to the active region between the coronal hole and the footpoint of the Helios trajectory and 


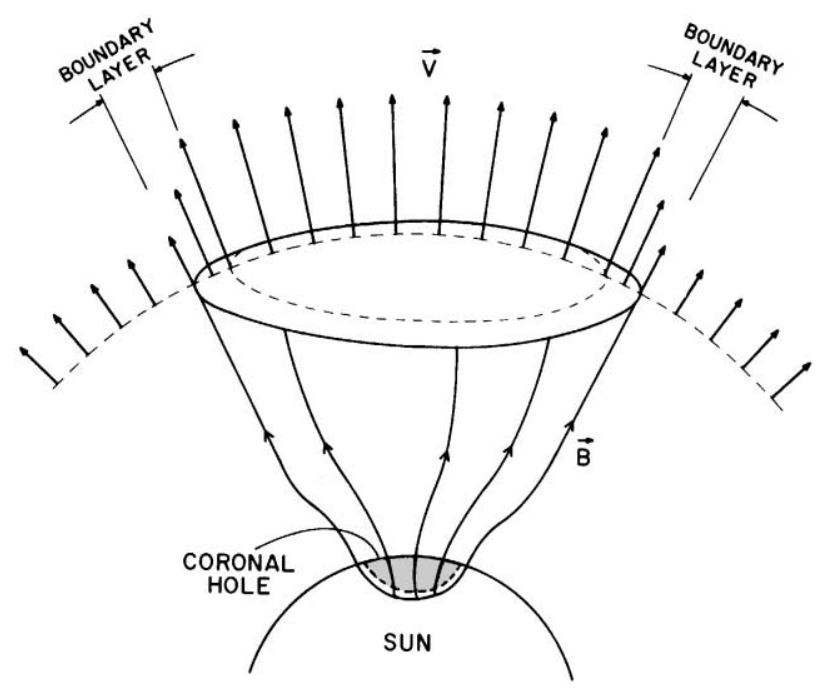

Fig. 6. Sketch of the divergence of streamlines and magnetic field line emanating from a coronal hole near the Sun, illustrating the expansion and boundary layer.

to the relatively large latitudinal separation between Helios and the coronal hole.

Fig. 5 shows that Helios 1 observed the second corotating stream even though the spacecraft passed several degrees below the coronal hole that was its source. Moreover, the plasma data in Fig. 5 show that the longitudinal boundaries of the streams were sharp near $0.3 \mathrm{AU}$ but not discontinuous. These results suggest that there is some divergence of the flow from the coronal hole, as illustrated in Fig. 6. This figure assumes that the divergence occurs in a boundary layer at the edge of the coronal hole. At the front boundary of the stream A3 in Fig. 5 the speed increased from 345 to $625 \mathrm{~km} / \mathrm{s}$ over an angle $\delta^{+}=2.1^{\circ}$. Since the velocity and magnetic field were nearly radial, this implies a velocity shear $>130 \mathrm{~km} / \mathrm{s} \mathrm{deg}^{-1}$. At the rear boundary of the stream the speed decreased from 625 to $345 \mathrm{~km} / \mathrm{s}$ over an angle of $\delta^{-}=12.3^{\circ}$, corresponding to a velocity shear of $\geqslant 20 \mathrm{~km} / \mathrm{s} \mathrm{deg}^{-1}$. The front boundary is thinner than the rear boundary at Helios, because the fast flow in the middle of the stream overtakes the slower flow at the front boundary and runs away from the slower flow at the rear boundary. The kinematic steepening at the front of the stream compresses the material between the slow and fast flow and produces the peaks in $B$ and $n$ that are evident in Fig. 5. Burlaga et al. (1978) unfolded the kinematic steepening effect, neglecting dynamical effects, and found that at $2.5 \mathrm{Rs}$ the width of the front and rear boundary was $\delta^{+}=11.8^{\circ}$ and $\delta^{-}=2.9^{\circ}$, respectively. Owing to the neglect of dynamical effects $\delta^{+}$is a lower limit and $\delta^{-}$is an upper limit, so that the best estimate of the latitudinal width of the boundary of the corotating stream at $2.5 \mathrm{Rs}$ was $7.4 \pm 4.5^{\circ}$.

The latitudinal width of the boundary of the corotating stream observed by Helios 1 in mid-March, 1975 was discussed by Schwenn et al. (1978) using speed profiles measured at 1 AU by Imp $7 / 8$ and near $0.3 \mathrm{AU}$ by $\mathrm{He}-$ lios 1 and coronal hole data determined from coronagraph observations. Imp 7/8 observed a single broad corotating stream associated with the coronal hole, whereas Helios 1 observed two narrow corotating streams separated by slow flow, as discussed above. This difference was interpreted by Schwenn et al. (1978) as a latitudinal gradient in the speed of the corotating stream, because the latitudes of IMP $7 / 8$ and Helios 1 were $\approx 5^{\circ}$ South and $\approx 5^{\circ}$ North, respectively, at the times they observed the fast flows. Thus, Schwenn et al. (1978) conclude that the latitudinal width of the northern boundary of the stream between $\approx 0.31$ and $1 \mathrm{AU}$ was $<10^{\circ}$, corresponding to a latitudinal shear of $\geqslant 30 \mathrm{~km} / \mathrm{s} \mathrm{deg}^{-1}$ in that region. These numbers for the boundary width and latitudinal speed gradient between 0.3 and $1 \mathrm{AU}$ are comparable to those derived for $0.3 \mathrm{AU}$ by Burlaga et al. (1978).

Evidence for large latitude gradients in the corotating stream speed profiles was found in other Helios observations. Schwenn (1990, p. 121) gives an example of a latitudinal gradient of $\left.(250 \mathrm{~km} / \mathrm{s}) / 1.5^{\circ}\right)$. Boundaries of $\approx 5^{\circ}$ width were observed out to $5 \mathrm{AU}$ (Mitchell et al., 1981). Ulysses (McComas et al., 1998) also measured a large latitudinal gradient of the speed at the boundary of the corotating stream that originated from a polar coronal hole.

Another interpretation of the difference between the speed profile observed by IMP $7 / 8$ at $1 \mathrm{AU}$ and that observed by Helios 1 near $0.3 \mathrm{AU}$ is the following. The coronal hole observations in Fig. 5 suggest that the two corotating streams observed by Helios 1 near 0.3 AU originated in two different coronal holes. The first stream in Fig. 5 was caused by the equatorial extension of a lobe of the south polar coronal hole, and the second stream was caused by a near-equatorial coronal hole at higher latitudes, as discussed above. If this is the case, then the single broad stream observed by IMP $7 / 8$ at 1 AU could have been caused by the merging of the two streams observed by Helios 1 .

An important conclusion is that corotating streams interacting with Mercury can produce a large and variable input signal to its magnetosphere. The input signal at Mercury is stronger and more variable than that at Earth, because the flows at Mercury are younger and closer to the source. Interactions among flows between the orbits of Mercury and Earth cause loss of memory of the details of the solar sources.

\section{Magnetic clouds and other ejecta}

It has been known for decades that in addition to the quasi-steady corotating streams from coronal holes, there are transient flows in the interplanetary medium (ejecta) associated with impulsive events on the Sun (Hundhausen, 1972; Burlaga, 1995). One class of ejecta that is particularly effective in influencing the magnetic field and magnetosphere of Earth and presumably of Mercury as well is the set 


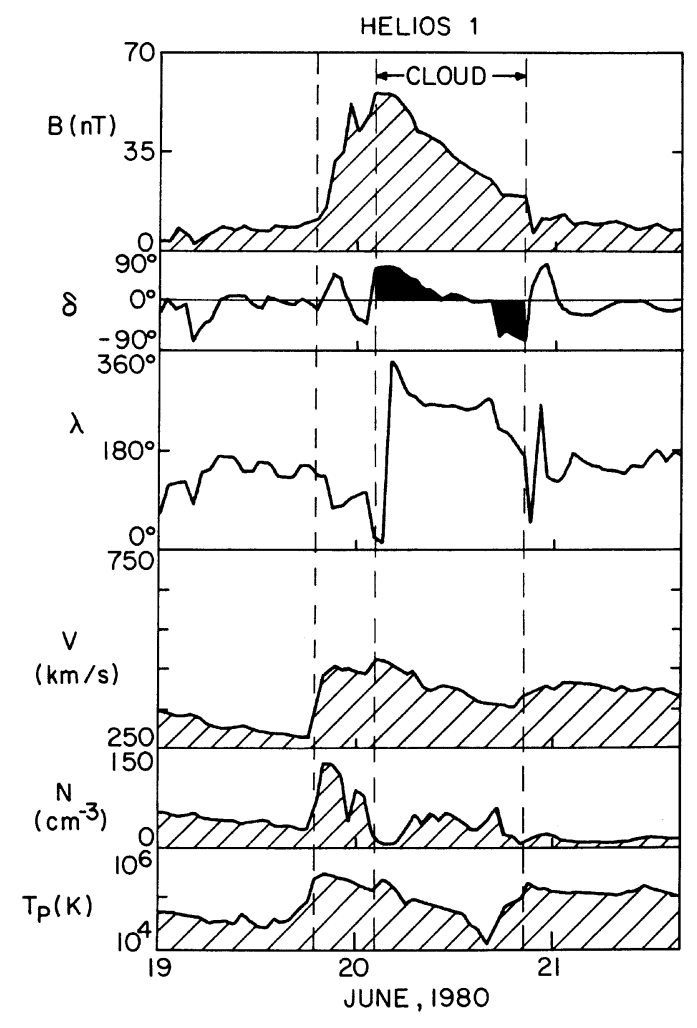

Fig. 7. Observations of a magnetic cloud by Helios 1 at $0.6 \mathrm{AU}$.

of magnetic clouds. A "magnetic cloud" is defined by three properties observed as it moves past a spacecraft at $\approx 1 \mathrm{AU}$ in a day or so: 1) a large, smooth rotation of the magnetic field direction; 2) higher than average magnetic field strengths; and 3) lower than average proton temperatures and $\beta$ (Burlaga et al., 1981). A magnetic cloud observed by Helios 1 on 20 June 1980 at 0.6 AU (Burlaga et al., 1982) is shown in Fig. 7. The magnetic field strength is high (maximum $\approx$ $60 \mathrm{nT}$ ), the direction rotates from a large northward direction to a large southward direction, and the proton temperature drops to $\approx 10^{4} \mathrm{~K}$ within the magnetic cloud. The speed decreases as the magnetic cloud moves past Helios 1, indicating that the magnetic cloud was expanding. The strong southward magnetic fields in such a magnetic cloud would produce substorms at Mercury, just as they do at Earth.

The geometry of a magnetic cloud was constrained by observations made by Helios 1 and 2, IMP 8, and Voyager 1 and 2 in January 1978, as illustrated in Fig. 8 (Burlaga et al., 1990). Burlaga et al. (1981) suggested that the magnetic field in a magnetic cloud has the form of a flux rope with possibly helical magnetic field lines anchored at the Sun. Goldstein (1983) proposed that the magnetic field $B$ in a magnetic cloud has the form of a structure known in physics as a force-free flux rope, in which $\boldsymbol{J}=\alpha \boldsymbol{B}$, where $\boldsymbol{J}$ is the current density and $\alpha$ is a function of position and time in general. Using the solution for a force-free field with constant $\alpha$ (the "Taylor state") and multi-spacecraft

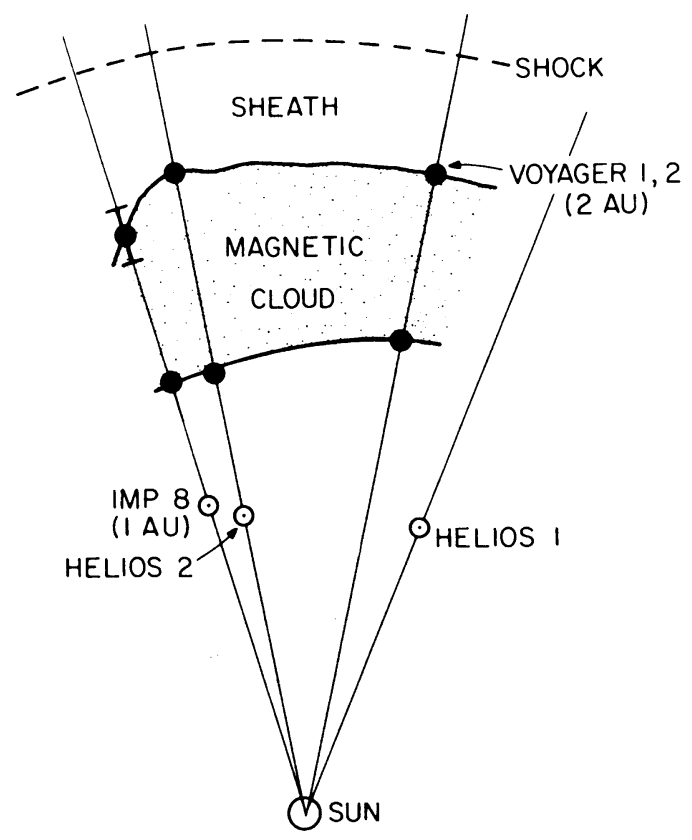

JAN 6,HR 22, 1978

Fig. 8. Boundaries of a magnetic cloud determined by observations from five spacecraft.

data, Burlaga et al. (1990) determined the geometry of the magnetic field in a magnetic cloud, which is illustrated in Fig. 9. Globally, the magnetic cloud has the form of a loop, and the magnetic field lines within the loop have the form of helices in a constant $\alpha$ force-free flux rope.

Magnetic clouds expand as they move away from the Sun, distributing magnetic energy over a larger volume with increasing distance. As a result, the magnetic field strength in a magnetic cloud is stronger at Mercury than at Earth. This effect is in addition to the increase in field strength with decreasing distance from the Sun corresponding to the Parker field, which is a result of the spherical solar wind expansion and solar rotation. Thus, the magnetic field strength and magnitude of the north-south component of the magnetic field will generally be several times stronger at Mercury than at Earth. Magnetic clouds generally produce relatively strong magnetic storms at Earth. Since magnetic storms are driven largely by the southward component of the magnetic field at both Earth and Mercury, one expects that the storms and substorms produced by magnetic clouds to be more intense at Mercury than at Earth.

\section{Interacting flows and energetic particles}

The largest values of the physical quantities that govern the interaction between the solar wind and Mercury are produced by interactions among flows and shocks. Fig. 10 shows one configuration that was studied in detail with observations from Helios 1, Helios 2 and IMP at Earth 


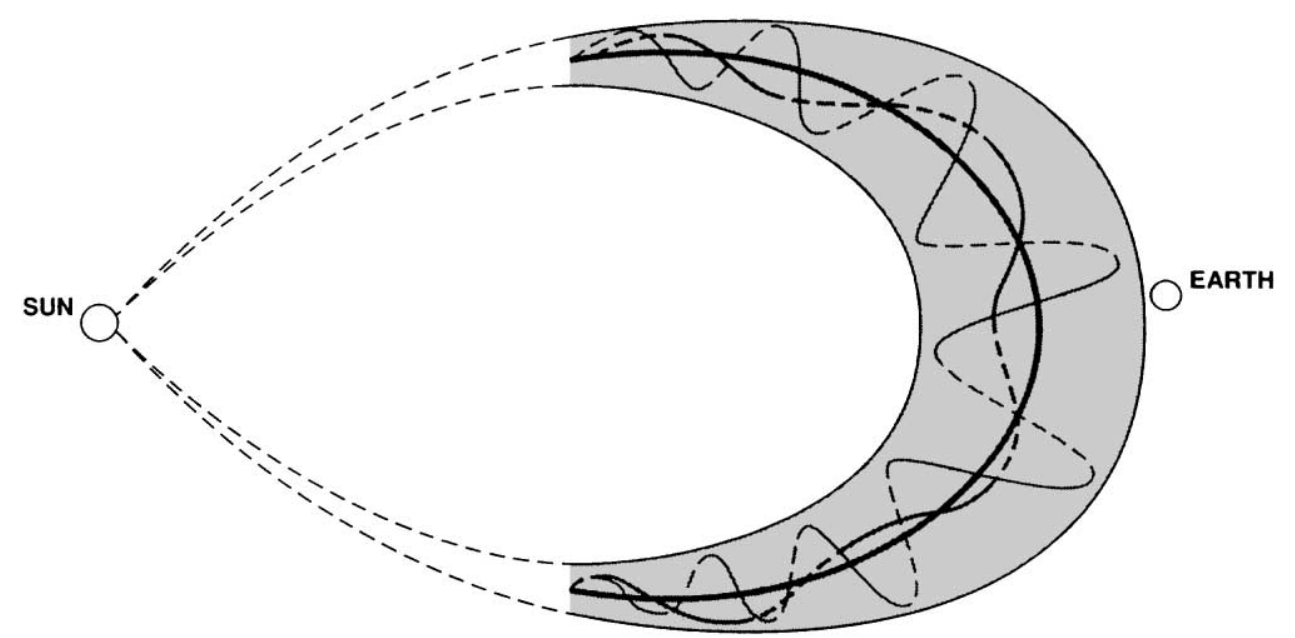

Fig. 9. Sketch of the geometry of a magnetic cloud and the magnetic field lines within the magnetic cloud. The magnetic field lines are helical, and they are shown here in projection. The connectivity of the field lines to the Sun is not fully understood, as indicated by the dashed lines near the Sun.

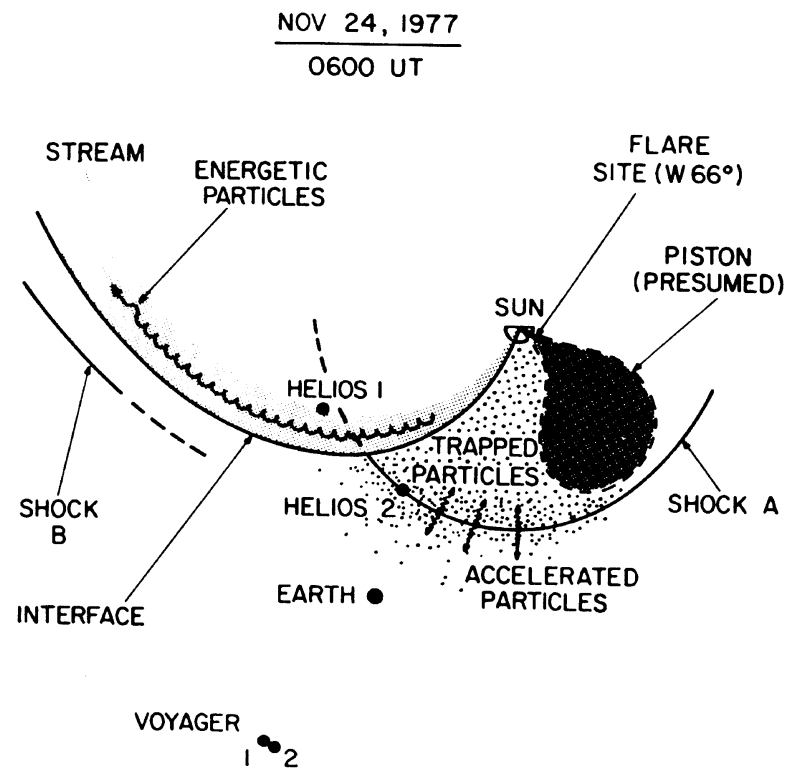

Fig. 10. Magnetic clouds (and other ejecta) and shocks driven by magnetic clouds can interact with other flows such as a corotating stream as illustrated here. The interaction can intensify the magnetic fields and density, creating conditions for a stronger interaction between the solar wind and Mercury than the flows could produce individually. The shocks accelerate particles, which can be trapped between two types of flows as shown here.

(Burlaga et al., 1980). The reconstruction shows the essential features of the flows at 0600 UT on 24 November 1977. A transient shock (shock A) was observed by Helios 2 and at Earth. It is assumed that such shocks are driven by ejecta, which act as a piston in analogy with laboratory studies of shock waves. In this case, the ejecta were not observed directly, but it is likely that the ejecta were associated with a flare at $\mathrm{W} 66^{\circ}$; hence the presumed ejecta are sketched by dark area west of the Earth-Sun line in
Fig. 10. A corotating stream was also moving through the solar wind, and Helios 1 was immersed in this flow. The front of a corotating stream within $1 \mathrm{AU}$ is identified by a thin stream interface. The stream interface is represented as a surface whose intersection with the ecliptic is a spiral, shown by a solid curve in the figure. The corotating stream drove a corotating shock (shock B) that formed beyond $1 \mathrm{AU}$ and was observed by Voyagers 1 and 2. The piston driven shock $A$ interacted with the corotating stream and its interaction region, enhancing the already strong fields in the corotating interaction region. However, a part of the shock was weakened as it passed through the corotating interaction region, and Helios 1 did not observe the shock.

From Fig. 10 one can readily imagine other types of interactions that can occur and increase the magnitude of the quantities that influence the interaction between the solar wind and Mercury. For example, a shock driven by another ejection or by a corotating stream could enter a magnetic cloud, thereby further increasing the relatively strong magnetic fields in the magnetic cloud. A corotating stream might overtake a magnetic cloud, compressing both the magnetic cloud and the corotating interaction region. One ejecta might overtake another, resulting in a compression in both ejecta. And so on.

Interplanetary shocks accelerate protons up to energies of the order of 1-10 MeV. Protons with energies of the order of $100 \mathrm{KeV}$ are accelerated abundantly. Particles with these energies are also accelerated by substorms at Mercury (Russell et al., 1988), and they can penetrate to the surface of Mercury. The intensity of such solar energetic particles is several times greater at Mercury than at Earth. The intensity depends on the evolving solar wind configurations. For example, in the configuration illustrated in Fig. 10, the particles accelerated by the shock were trapped between the stream interface, the ejecta, and the 


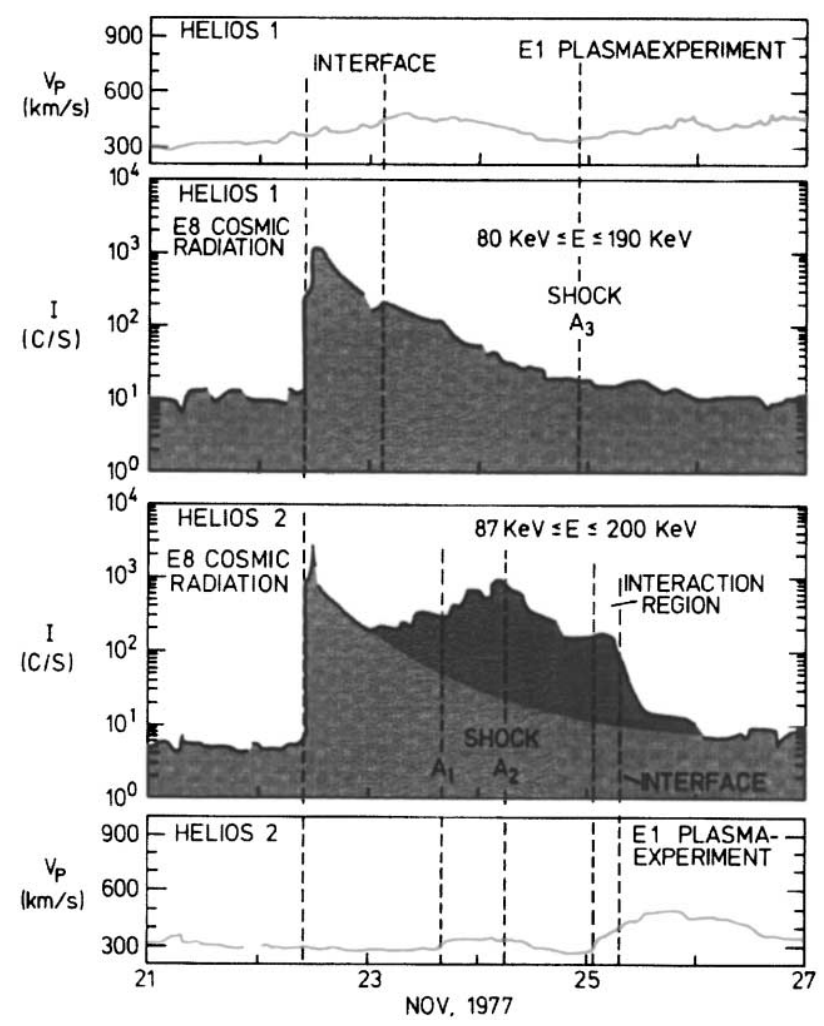

Fig. 11. Observations of the speed and energetic particles by Helios 1 and 2 for the configuration shown in Fig. 10.

shock, resulting in relatively high intensities there (Burlaga et al., 1980). Fig. 11 shows that particles with energies $80 \mathrm{KeV}<E<200 \mathrm{KeV}$ had a local maximum intensity at the shock seen by Helios 2 and remained intense until the stream interface arrived, after which the intensity decreased abruptly by an order of magnitude there. Thus, the stream interface was a relatively impermeable barrier to the shock accelerated particles. Helios 1 , which was located at 0.6 AU inside the corotating stream, did not observe the shock. Helios 1 observed the solar energetic particles arrive abruptly, but then the particles decayed monotically with increasing time as the particles moved rapidly away from the Sun on the spiral magnetic field lines in the corotating stream.

Energetic electrons are accelerated at the Sun and in the magnetosphere of Jupiter, and they are rapidly transported along spiral field lines. When spiral field lines connect the magnetospheres of Jupiter and Mercury, they can channel energetic electrons from Jupiter to the surface of Mercury.

Clearly, the interactions among different types of flows and shocks can influence strongly the parameters that control the size and activity of magnetosphere of Mercury and the structure of its magnetic field. The flow configurations and shocks also influence the distribution and motions of the energetic particles that can enter the magnetosphere of Mercury and reach its surface. Knowledge of these flows and energetic particles is essential to understanding Mercury itself.

\section{Summary}

The solar wind and energetic particles interact with Mercury's planetary magnetic field and magnetosphere, and they can also interact directly with the surface of Mercury. Variations of the solar wind parameters and the interplanetary magnetic field will produce constantly changing configurations of Mercury's global magnetic field and substorm processes in Mercury's magnetosphere. Particles accelerated in such substorms can carry significant amounts of power to the dark side of Mercury producing surface heating in the auroral zones (Russell et al., 1988). For these and other reasons, an understanding of the solar wind and energetic particle environment of Mercury is essential for interpreting the observations from a Mercury orbiter.

Helios 1 and 2 provided us with some samples of the environment of Mercury from 1974 to 1986 . We reviewed some of the basic characteristics of the solar wind in this region. The most important point is that the solar wind will interact much more strongly with Mercury than it does with Earth, because the parameters that govern the interaction are much larger (by approximately an order of magnitude) at Mercury than at Earth. This interaction is highly variable because the solar wind itself is highly variable in Mercury's orbital zone. Our knowledge and understanding of this variability is limited. Further studies of the environment of Mercury based on the Helios data should be carried out to provide additional knowledge that will be useful for interpreting the results of the Mercury orbiters. However, that knowledge will still be limited, owing to the large variety of configurations that can occur. The variable environment of Mercury will strongly affect Mercury's magnetic field, magnetosphere and even its surface. In order to derive the most from the observations of Mercury, it is very important to take every opportunity to measure the solar wind by the Mercury orbiters themselves, as they move to Mercury and in orbit about Mercury.

\section{References}

Baker, D.N., Akasofu, S.-I., Baumjohann, W., Bieber, J.W., Fairfield, D.H., Hones, E.J. Jr., Mauk, B., McPherron, R.L., Moore, T.E., 1984. Substorms in the magnetosphere. In: Butler, D.M., Papadopoulos, K. (Eds.), Solar Terrestrial Physics: Present and Future, NASA Reference Publication 1120, Washington DC.

Bougeret, J.L., King, J.H., Schwenn, R., 1984. Solar radio burst and in situ determination of interplanetary electron density. Sol. Phys. 90 (2), 401-412.

Burlaga, L.F., 1974. Interplanetary stream interfaces. J. Geophys. Res. $79,3717$.

Burlaga, L.F., 1991. Magnetic Clouds. In: Schwenn, R., Marsch, E. (Eds.), Physics of the Inner Heliosphere II. Springer, Berlin, pp. 1-19.

Burlaga, L.F., 1995. Interplanetary Magnetohydrodynamics. Oxford University Press, New York.

Burlaga, L.F., Ness, N.F., Mariani, F., Bavassano, B., Villante, U., Rosenbauer, H., Schwenn, R., Harvey, J., 1978. Magnetic fields and flows between 1 and 0.3 AU during the primary mission of Helios 1 . J. Geophys. Res. 83 (A11), 5167-5174.

Burlaga, L.F., Lepping, R., Weber, R., Armstrong, T., Goodrich, C., Sullivan, J., Gurnett, D., Kellogg, P., Keppler, E., Mariani, F., 
Neubauer, F., Rosenbauer, H., Schwenn, R., 1980. Interplanetary particles and fields, November 22 to December 6, 1977: Helios, Voyager and Imp observations between 0.6 and 1.6 AU. J. Geophys. Res. 85, 2227.

Burlaga, L.F., Sittler, E., Mariani, F., Schwenn, R., 1981. Magnetic loop behind an interplanetary shock Voyager, Helios and IMP-I observations. J. Geophys. Res. 86, 6673.

Burlaga, L.F., Klein, L., Sheeley, N.R., Michels, D.J., Howard, R.A., Koomen, M.J., Schwenn, R., Rosenbauer, H., 1982. A magnetic cloud and a coronal mass ejection. Geophys. Res. Lett. 9 (12), 1317-1320.

Burlaga, L.F., Lepping, R., Jones, A.P., 1990. Global configuration of a magnetic cloud. In: Russell, C.T., Priest, E.R., Lee, L.C. (Eds.), Physics of Magnetic Flux Ropes. American Geophysical Union, Hamilton, Bermuda, pp. 365-378.

Connerney, J.E.P., Ness, N.F., 1988. Mercury's magnetic field and interior. In: Vilas, F., Chapman, C.R., Matthews, M.S. (Eds.), Mercury. The University of Arizona Press, Tucson, pp. 494-593.

Denskat, K.U., Neubauer, F.M., 1982. Statistical properties of low-frequency magnetic field fluctuations in the solar wind from 0.29 to $1.0 \mathrm{AU}$ during solar minimum conditions-Helios-1 and Helios-2. J. Geophys. Res. 87 (A4), 2215-2223.

Goldstein, H., 1983. On the field configuration in magnetic clouds. In Neugebauer, M. (Ed.), Solar Wind Five. NASA Conference Publishing, NASA CP-2280, 731-733.

Hundhausen, A.J., 1972. Coronal Expansion and Solar Wind. Springer, New York.

Hundhausen, A.J., 1977. An interplanetary view of coronal holes. In: Zirker, J.B. (Ed.), Coronal Holes and High Speed Wind Streams. Colo. Association University Press, Boulder, Co, pp. 225-329.

Kunow, H., Wibberenz, G., Green, G., Muller-Mellin, R., Kallenrode, M.-B., 1991. Energetic particles in the inner solar system. In: Schwenn, R., Marsch, E. (Eds.), Physics of the Inner Heliosphere II. Springer, Berlin.

Mariani, F., Ness, N.F., Burlaga, L.F., Bavassano, B., Villante, U., 1978. Large-scale structure of interplanetary magnetic field between 1 and 0.3 AU during the primary mission of Helios-1. J. Geophys. Res. 83 (A11), 5161-5166.

Mariani, F., Neubauer, F.M., 1990. The interplanetary magnetic field. In: Schwenn, R., Marsch, E. (Eds.), Physics of the Inner Heliosphere I. Springer, Berlin, pp. 183-205.
Marsch, E., 1991. MHD turbulence in the solar wind. In: Schwenn, R., Marsch, E. (Eds.), Physics of the Inner Heliosphere II. Springer, Berlin, pp. 159-235.

Marsch, E., Mulhlauser, K.-H., Schwenn, R., Rosenbauer, H., Pilipp, W.G., Neubauer, F.M., 1982. Solar wind protons: Three dimensional velocity distributions and derived plasma parameters, 0.3 and $1.0 \mathrm{AU}$. J. Geophys. Res. 87, 52-72.

McComas, D.J., Riley, P., Gosling, J.T., Balogh, A., Forsyth, R., 1998. Ulysses' rapid crossing of the polar coronal hole boundary. J. Geophys. Res. 103 (A7), 14,655-14,655.

Mitchell, D.G., Roelof, E.C., Wolfe, J.H., 1981. Latitude dependence of solar wind velocity observed $>1$ AU. J. Geophys. Res. 86, $165-179$.

Musmann, G., Neubauer, F.M., Lamers, E., 1977. Radial variations in the interplanetary magnetic field between 0.3 and $1.0 \mathrm{AU}$ : Observations by Helios 1. J. Geophys. Res. 82, 551-562.

Ness, N.F., Behannon, K.W., Lepping, R., Whang, Y.C., Schatten, K.H., 1974. Magnetic field observations near Mercury: Preliminary results from Mariner 10. Science 185, 151-160.

Ogilvie, K.W., Scudder, J.D., Hartle, R., Siscoe, G.L., Bridge, H.S., Lazarus, A.J., Asbridge, J.R., Bame, S.J., Yeates, C.M., 1974. Observations at Mercury encounter by the plasma science experiment on Mariner 10. Science 185, 146-152.

Parker, E.N., 1963. Interplanetary Dynamical Processes. Interscience Publishers, New York.

Russell, C.T., Baker, D.N., Slavin, J.A., 1988. The magnetosphere of Mercury. In: Vilas, F., Chapman, C.R., Matthews, M.S. (Eds.), Mercury. The University of Arizona Press, Tucson, pp. 514-561.

Schwenn, R., Montgomery, M., Rosenbauer, H., Miggenreiter, H., Bame, S., Feldman, W., Hansen, R.T., 1978. Direct observations of the latitudinal extent of a high-speed stream in the solar wind. J. Geophys. Res. 83, 1011.

Schwenn, R., 1990. Large-scale structure of the interplanetary medium. In: Schwenn, R., Marsch, E. (Eds.), Physics of the Inner Heliosphere I99-171. Springer, Berlin.

Slavin, J.A., Holzer, R.E., 1981. Solar wind flow about the terrestrial planets: 1 . Modeling bow shock position and shape. J. Geophys. Res. $86,11,4041-11,408$.

Whang, Y.C., 1977. Magnetospheric magnetic field of Mercury. J. Geophys. Res. 82, 1024-1030. 